\title{
COLLABORATIVE SERVICES TO MAINTAIN ELECTRONIC BUSINESS RELATIONSHIPS
}

\author{
Peter Weiß, Stefan Klink \\ Institute of Applied Informatics and Formal Description Methods (AIFB), \\ University of Karlsruhe (TH), GERMANY \\ \{Peter.Weiss, Stefan.Klink\}@aifb.uni-karlsruhe.de
}

\begin{abstract}
Electronic collaborative networks are a prevailing concept in actual scientific business management literature. Because of the occurrence of newly concepts as "service orientation" and service oriented architectures, electronic networks and business intelligence, has gained momentum and revival. In the paper we look at the conceptual design of a framework for the development of collaborative services to maintain electronic business relationships. The reader gets acquainted with relevant theories and research strands that need to flow into a design framework of collaborative services.
\end{abstract}

\section{INTRODUCTION}

During the last decade, along with growing interest and increasing use of electronic networks, society, science and business have been affected by remarkable changes. Electronic networks have had a tremendous impact on the every day and working life changing the way people interact, live and work. In our economy today, networking plays a crucial role in various branches and application domains though there are still numerous barriers to overcome. In this connexion collaborative services are a new paradigm likely to further leverage collaboration and networking among agents, whether humans or machines, in electronic networks.

In focus of this research are social ties between network entities. In electronic networks the linkages of entities (e.g. business partners, employees, experts, etc.) have to be supported by electronic services. Services are designed to overcome identified boundaries for Collaborative Networks (Martin-Flatin et al., 2006). Semantics, self-organisation, security, trust and privacy, awareness and incentives are variables influencing the relational ties between units. However, linkages between agents require the right climate and conditions of a collaboration environment. This environment should provide opportunities for or constraints on individual action in a specific given context. These horizontal dimensions facilitate and catalyse the emergence of relational processes and structures to evolve.

First, the paper overviews briefly the current state of the art of collaborative networks with clear focuses on the aspects of business relationship management. The authors motivate their research by explaining the vision and related challenges of dynamic business ecosystems.

Wciß, P., Klink, S., 2007, in IFIP Intcrnational Fedcration for Information Processing, Volume 243, Establishing the Foundation of Collaborative Networks; eds. Camarinha-Matos, L., Afsarmanesh, H., Novais, P., Analide, C.; (Boston: Springer), pp. 435-442. 


\section{COLLABORATIVE NETWORKS SO FAR}

Today, there is a growing community of researchers whose work gravitates towards the topic of Collaborative Networks (Camarinha-Matos et al., 2003, 2004, 2006). The research community has elaborated in the meanwhile a comprehensive knowledge base of Collaborative Networks encompassing theoretical foundations, services, collaboration and innovation environment, and last but not least the analysis of the impact collaborative networks have on daily life and economy. The need of a comprehensive scientific framework of collaborative networks (see Figure 1) is argued facilitating to structure and to align interdisciplinary research activities towards common goals and visions.

From a network perspective, linkages and related relational processes and structures are the subject of analysis. The proposed framework allows to analyse and to conceptualise structures in form of models but also considers the patterns of emerging and evolving relationships among agents (human and machines). Subject of analysis are related interactions at the level of individuals, teams (or groups), organisations, networks and communities. It supports the evolution of dynamic networks and the self-organisation of network entities. The required emergence of relational structures requires specific services within collaborative networks.

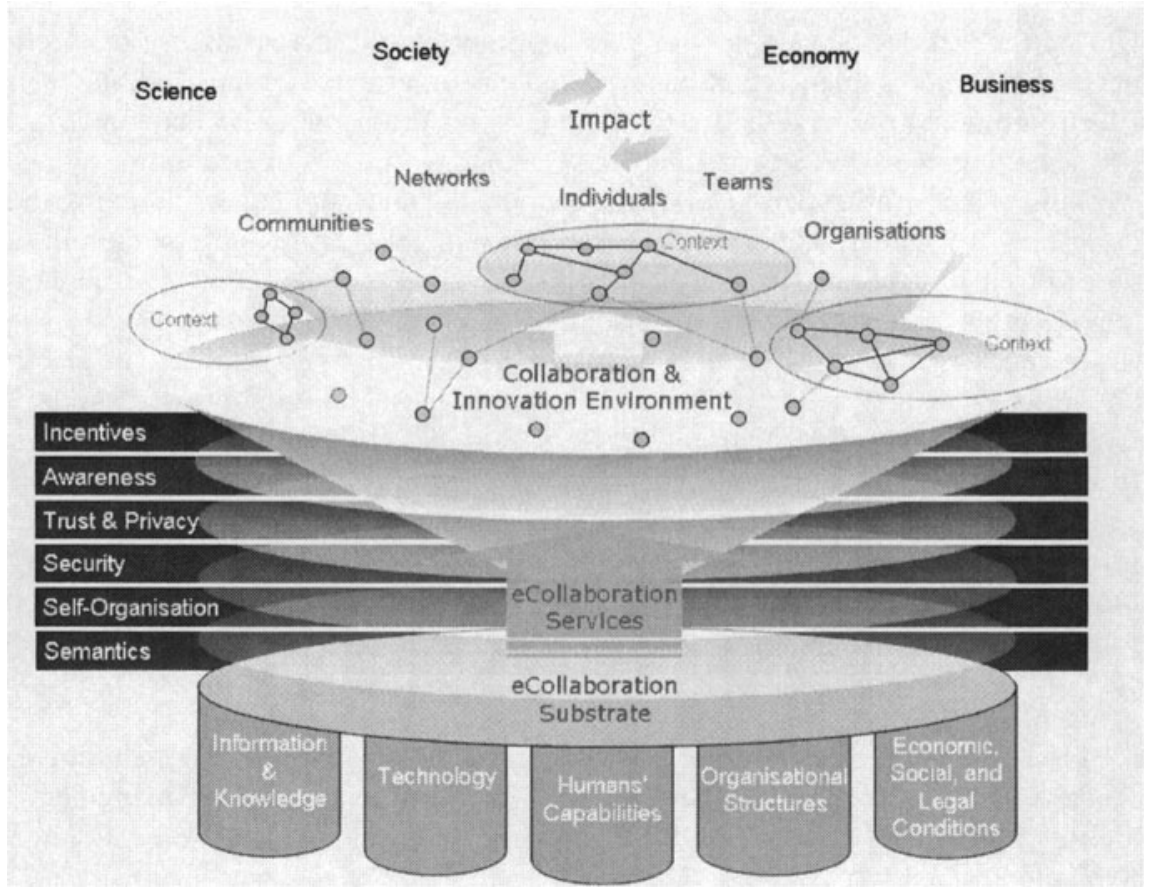

Figure 1 - Framework of collaborative networks' infrastructure

\section{OBJECTIVES}

Our research thesis claims that organisational structures with demanded abilities to self-manage, self-configure and self-optimise require besides the necessary culture 
"[...] semantic-informed self-organizing structures [...]" (Camarinha-Matos et al., 2003 , p. 8). Culture and structure are constituted in the collaboration and innovation environment and provides the "breeding environment" for establishing relational ties between agents (e.g., units, actors, etc.). From a network perspective, linkages and related relational processes and structures are subject of analysis. The concept of semantically enriched business partner profiles and sound, proven techniques of information retrieval, data mining, and machine learning, respectively, are combined together to develop the necessary collaborative services environment. In our focus are discovery and matching services respectively because they provide the field for further enhanced services and functionality in collaborative networks.

\section{REVIEW OF STATE-OF-THE-ART}

\subsection{Social Networks and Analysis}

The theoretical part is based on the theory of social networks (Wassermann, Faust 1994). Due to the fact that business relationships contains not only people but resources of any kind (material or nonmaterial) common one-mode dyadic networks are not sufficient to represent the given network structure. In our case, we need so called multi-mode social networks described in the following paragraphs.

\section{Basics of Social Networks}

A social network can be seen as a graph $G=(V, E)$ in which the vertices $V$ represent the actors, the edges $\mathrm{E}$ the ties between these actors. Clustering describes the situation that if one actor has a tie (of arbitrary origin) to two other actors then it is likely that these two individuals will start a tie as well. This can easily be seen while looking at friends one has and then at the friends they have. It is likely, as Watts (2004) points out that there will be a certain overlap between these two sets of people. If the clustering is advanced and the characteristic path length in a social network satisfies certain conditions, then the observed social network is a specific subtype of network, a so called small world network. In the case of business partners such a small world network is the basis of new algorithms for similarity measurement. The network considered is the business partner network constructed from the database of business partner profiles. The actors are the partners listed in the dataset. A tie between two business profiles is inserted into the graph if the actors are collaborating. The usage of such networks in general is not new in order to find synonyms. However the manner of which and how this information is used is innovative.

\section{Multi-mode Social Networks}

For representing additional information multi-mode networks are needed. In contrast to common one-mode networks with persons as actors and friendship as relation, multi-mode networks are capable to represent relationships of various types. These kinds of networks are also known as affiliation or membership networks where one set of actors (here: business partners) and multiple sets of events (here: contracts, employments, deliverers, developers etc.) are present (Wassermann, Faust, 1994, Chapter 8). Relationships within business partners are manifold and can be listed as a hierarchy with increasing indirection in within several levels. 
Social Software \& Web 2.0

Social Software supports, extends, or derives added value from human social behaviour (Coates, 2005). It can be used not only for information and identification management but also for relationship management. Social software, e.g. Blogs, Wikis, message boards, social bookmarking/tagging and navigation etc., support to map and to foster current contacts and to endeavour and to tie new contacts. The focus on the web 2.0 lies on the exchange, interaction, and (re)usability of information. It is an easy to use platform for social and collaborative exchange of general information, news, data and services. Web 2.0 applications manipulate the content, the structure and the layout of documents and present all the information in a new combination more or less condensed to the user.

\subsection{Business Partner Profiles}

The selection of business partners can be identified as key to success and key capability for the realisation of dynamic business ecosystems in near future. Thus, there is a need to conceive business relationships as intangible asset of an enterprise that needs special care. Namely, relational ties are to be maintained continuously through an adequate management. Weiß (2005) proposed a newly approach for business partner management with ontologies in large business communities. This research strived to leverage the capabilities to cope with large numbers of business relationships. New approaches are required to expand the boundaries of electronic networks. A possible solution seems to be support through appropriate services of the ICT infrastructure (Martin-Flatin, 2006). Ontologies offer support for communication processes and complex interactions of business entities in collaborative spaces (Weiß, 2007). The analysis of business relationships and related interactions requires an appropriate analysis framework that allows to investigate and to describe the nature of relational structures and processes.

\subsection{Information Retrieval}

In standard text information retrieval scenarios, textual queries are compared to a set of documents or a full-text index, respectively to get relevant documents. The same procedure is mostly done in multi-media retrieval for searching for images, videos or music. Recently research is trying to get more out of the query or to put more information into the query for enlargement or for making it more precise. Ontologybased systems try to categorize the users query into a specific ontology entity and then the query is enriched with information stored in the ontology (Klink, 2006).

Another information retrieval approach is to search for similar entities, i.e. not a manually formulated query is given by the user but the user selects an appropriate document, image or piece of music as a query and then the IR-System compares this with all others. More or less sophisticated ranking methods are sorting the results or clustering algorithms are used to categorize the results into various topics. Approaches in the field of Case-Based-Reasoning (CBR) are working analogously. The current (new) case is matched to (known) cases stored in a database and most similar (best matching) cases are retrieved (Bergmann, 2002). In our framework, ontology-based as well as similarity-based approaches are used for discovery and/or matching the BPP mentioned above. Instead of querying business partners by manually formu- 
lated queries, our framework is capable for more sophisticated approaches described below.

\subsection{Trust in Electronic Networks}

Obviously, the increasing dynamics in the interaction of business partners combined with the shortening life-cycle of co-operations contradict the nature and actual needs of business relationships regarding trust and commitment. Both concepts occur over a longer period of time and require stable relationships. Once trust is established it reduces opportunistic behaviour. This is substantiated by existing organisation theories as e.g. transaction cost, principal-agent and property-rights (Picot et al., 2003, p. 45ff.), (Sydow, 1992, p. 130ff.). Trust is the base for a successful business relationship and therefore essential for the success of business endeavours within selfforming networks. The success of virtual organisations depends largely on quality of relational ties between network members. As in social software building and maintaining trust is essential to reduce opportunistic behaviour. Legal contracts might never be totally disappear in real business life though increasing respective transaction costs. Although reducing network efficiency contracts are an appropriate mean to increase the "intensity of linkage" of network structures. Accordingly, advanced collaborative services (e.g. filtering and pre-selection of business partners) aim at establishing zones of trust.

\section{CONCEPTUAL MODEL AND FRAMEWORK}

In the following, all approaches mentioned above are combined. A conceptual model is developed that serves the identified needs.

\subsection{Future of Digital Business Ecosystems}

In Figure 2 illustrates the three stages towards a digital business ecosystem. At stage one a centralised model for the business partner profiles (BPPs) is applied. The profiles represent information required to discover, select and integrate business partners preferably dynamic, on demand into emerging or existing value chains. The business partners that can be accessed through the ICT infrastructure are limited to the information stored and accessible through the registry (e.g. in form of an Enterprise Portal). Related work can be found in the area of business registries for web services as e.g. UDDI (Universal Description, Discovery and Integration) and ebXML (Electronic Business XML).

Current research gravitates around the realisation of the scenario shown at stage two. At this stage, a decentralised model is developed. BPPs are no longer stored centrally in an information portal. These profiles are stored decentralised but are created on basis of a shared conceptual model in form of a domain ontology. Business partners maintain latent business relationships solely within the boundaries of the business network they have registered to. The reach of the network are defined by the actual number of registered users.

Whereas, at stage three it is strived for an advanced decentralised model that allows to contact and query business partners as well outside the boundaries of the defined network. For the discovery and selection of business partners desired information needs to be retrieved beforehand through machines (e.g. through agents or 
web crawlers). Semantics of data stored in a machine-processable form in profiles have to be explicit. In this way, the described approach fulfils the requirements of described application scenario though it has to be further developed. Especially, retrieval of distributed information and heterogeneous data stored in complex data types (e.g. as elements of BPPs) poses interesting questions for future research endeavours (Weiß, 2007).

\subsection{Conceptual Framework}

In this section, the conceptual framework for the design of collaborative services to maintain electronic business relationships is looked at. Starting from the scenario one described in Figure 2 above, the aim is now to approach the visionary setting displayed at the next stages two and three.

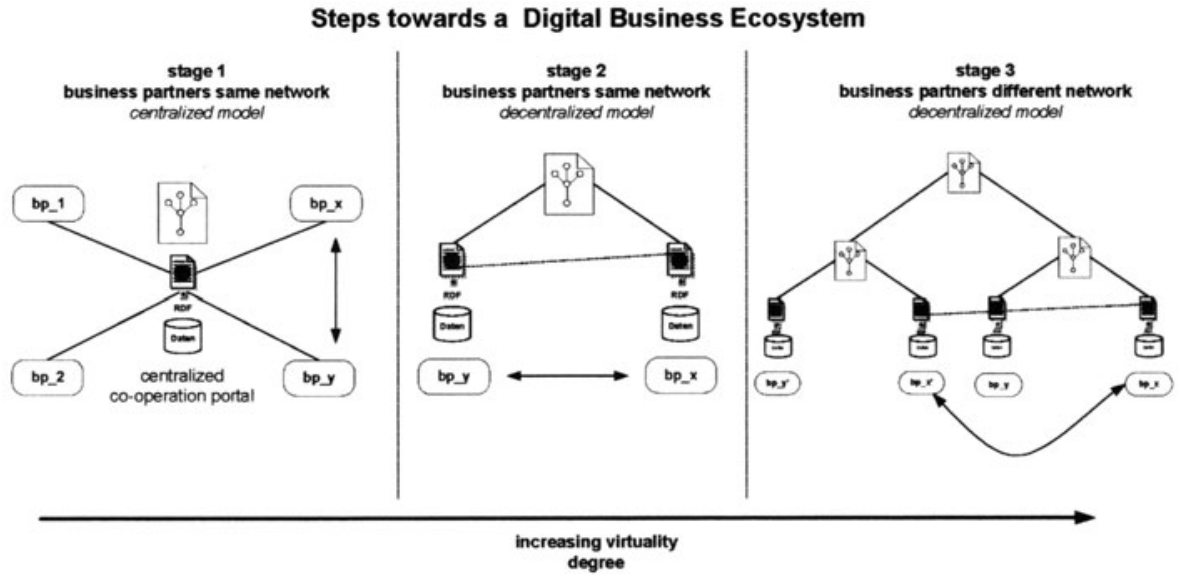

Figure 2 - Steps towards digital business ecosystems (Weiß, 2005)

The work follows the approach to assess decision criteria in order to support the self-formation and self-organisation of adaptive business networks. As a first step, a centralized approach with a shared explicit data model for BPPs as identity or individual "fingerprints" is planned. Profiles are based on an ontology-based model which has been derived from an empirical model for the network analysis encompassing important dimensions for the configuration and description of business relationships (Weiß 2007). BPPs are stored in a central or a distributed database for retrieval and for further analysis.

Complex data structures are required to express the complexity of and formalise business related information. They then can be accessed extracting relevant information from them within a specification of requirements (Field and Hoffner, 2003).

In summary, Figure 3 depicts the overall research approach. The configuration of the business relationship takes place primarily on the organisational layer. To involve the ICT infrastructure collaborative data has to be represented in machine-processable form. This can be realised using ontologies for the structuring and modelling of the BPP. Profiles are a prominent concept for example used by web services to 
store specified service descriptions (e.g. in WSDL (Web Services Description Language)).

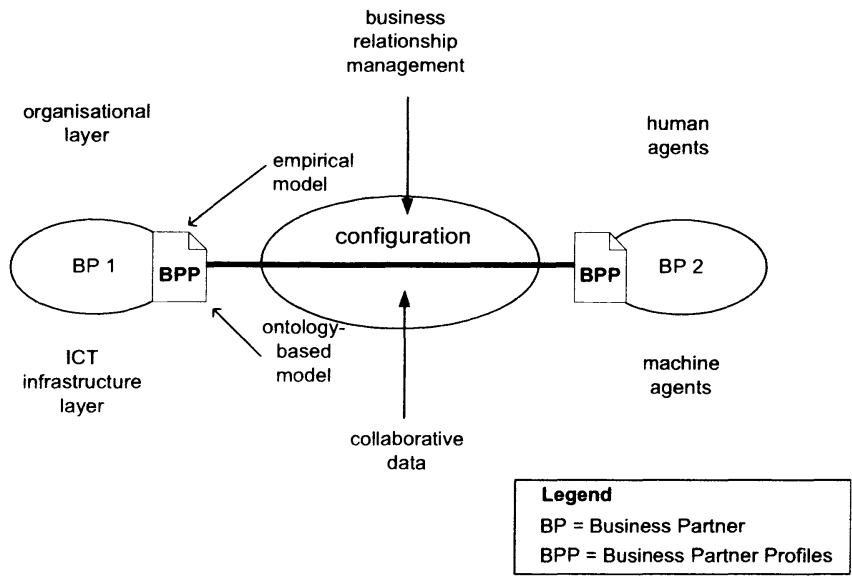

Figure 3 - Design of approach for business partner relationship management with ontologies (Weiß, 2007)

Therefore, this is seen as appropriate way to approach the problem at hand. BPP are anticipated to bridge the existing barrier between the organisational and ICT infrastructure layer. Through the application of ontologies, collaborative services as e.g. for discovery and/or matching of BPP can be used to support the self-reference and emergence of collaborative structure and cultures. Especially zones of trust can be established through filtering of collaborative information and an intensified interaction and information exchange of business entities in forefront of concrete business endeavours (Weiß, 2007).

\section{CONCLUSIONS AND OUTLOOK}

In the paper we followed the vision to support relational processes within electronic networks. Based on a review of existing state-of-the-art we presented pivotal theories, concepts and techniques that need to flow in an appropriate conceptual model of the management of business relationships. The combination of two strands: namely state-of-the-art data techniques (information retrieval, data mining, social network analysis, machine learning, etc.) and business partner profiling resulted into a novel framework. Though, the framework is not yet ready for implementation we have conceptualised all necessary components and have specified the needed functionality and technologies to develop a demonstrator. At the ICT infrastructure layer collaborative services, namely discovery and matchmaking services, are supposed to handle emergence and evolution of business relationships by processing of collaborative data. Respective data is stored in business partner profiles. Ontologies represent required semantics of data to achieve that both humans and machines can benefit and process the information sets. At the organisational layer the approach requires establishing an appropriate business relationship management. It encompasses mainly appropriate variables and selection criteria as well as a framework to configure respective parameters on which basis business relationships then likely are able 
to emerge and evolve. The goal is to realise the aspired strong interaction and linking of organisational and ICT infrastructure layer.

It has been pointed out, that presented research is still at stage of research design. Next steps to be taken are to choose and define complex data types in order to implement a prototype so that we gain a huge amount of real-live data for explorative research. On that basis, data techniques will be applied to analyse the data and to set up (personal) recommender supporting the Business Partners. Searching appropriate partners can be automized with agent technologies.

\section{REFERENCES}

1. Baeza-Yates R, Ribeiro-Neto B. Modern Information Retrieval. Addison-Wesley, 1999.

2. Bergmann R. Experience Management Foundations, Development Methodology, and InternetBased Applications. LNAI 2432, Springer Verlag, 2002.

3. Bhattacharya I, Getoor L. Iterative record linkage for cleaning and integration. In Proceedings of the 9th ACM SIGMOD Workshop DMKD 2004, Paris, France, June 13, 2004, pages 11-18. ACM, 2004.

4. Camarinha-Matos LM, Afsarmanesh H, Ollus M. Network-Centric Collaboration and Supporting Frameworks. IFIP TC5/WG5.5, Proceedings PRO-VE'06, 2006.

5. Camarinha-Matos LM. Virtual Enterprises and Collaborative Networks. IFIP TC5/WG5.5, Proceedings PRO-VE'04, 2004.

6. Camarinha-Matos LM, Afsarmanesh H. Processes and Foundations for Virtual Organizations. IFIP TC5/WG5.5, Proceedings PRO-VE'03, 2003.

7. Camarinha-Matos LM. Collaborative Business Ecosystems and Virtual Enterprises. IFIP TC5/WG5.5, Proceedings PRO-VE'02, 2002.

8. Field S, Hoffner Y. Web Services and matchmaking. In Intl. Journal Networking and Virtual Organisation, vol. 2, no. 1, 2003.

9. Martin-Flatin, Jean-Philippe; Sventek, Joe and Geihs, Kurt: Self-managed systems and services, in Communications of the ACM, March 2006, vol. 49, no. 3.

10. Klink S. Intelligent Query (Re-)Formulation with Concept-based Expansion. Universität Trier, Dissertation, Dr. Hut-Verlag, München, 2006.

11. Klink S. Improving Document Transformation Techniques with Collaborative Learned Term-based Concepts. LNCS 2956, pages 281-305, Springer, 2004.

12. Klink S, Kise K, Dengel A, Junker M, Agne S. Document Information Retrieval. In Chaudhuri BB, Digital Document Processing: Major Directions and Recent Advances, Springer, 2007.

13. Krystek U, Redel W, Reppegather S. Grundzïge virtueller Organisationen: Elemente und Erfolgsfaktoren, Chancen und Risiken. Wiesbaden, Gabler Verlag, 1997.

14. Picot A, Reichwald R, Wigand, RT. Die grenzenlose Unternehmung. Gabler Verlag, 2003

15. Scott J: Social Network Analysis. Second Edition. London: SAGE Publications Ltd, 2000.

16. Sydow J.: Strategische Netzwerke: Evolution und Organisation. Wiesbaden : Gabler Verlag, 1992.

17. Wasserman S, Faust K. Social Network Analysis: Methods and Applications. Cambridge University Press, 1994.

18. Watts DJ. Six Degrees: The Science of a Connected Age. W.W.Norton\&Company, New York, 2004.

19. Weiß P. Management von Geschäftsbeziehungen in virtuellen Organisationsstrukturen. Universität Karlsruhe (TH), Dissertation, Dr. Hut-Verlag, München, 2005.

20. Weiß P.: Towards Adaptive Business Networks: Business Partner Management with Ontologies. In Rittgen P, Handbook of Ontologies for Business Interaction, pp. 24; IDEA GROUP INC., 2007 (to appear). 